Accelerator Division

Alternating Gradient Synchrotron Department BROOKHAVEN NATIONAL LABORATORY

Upton, New York 11973

Accelerator Division

Technical Note

AGS/AD/Tech. Note No. 389

\title{
MeVVA SOURCE OF PRIMARY METALLIC IONS FOR SuperEBIS
}
A. Hershcovitch, A. Kponou, and
D. McCafferty

January 25, 1994

Introduction

Generation of high charge state heavy metallic ions including uranium has been successful in a number of EBIS (Electron Beam Ion Source) devices. The best results have been obtained in devices utilizing injection of primary, low charge state, ions from an external ion source into the EBIS trap for further ionization into higher charge states. In one such device, named EBIT (Electron Beam Ion Trap), a MeVVA ion source is used as an injector of primary metallic ions into the EBIT trap. We fabricated and experimented with a MeVVA metallic ion source. This source was recently installed on SuperEBIS, and has successfully injected titanium and uranium ions into the SuperEBIS trap.

\section{General MeVVA Description}

MeVVA (which is the acronym from metal vapor vacuum arc) is a prolific generator of highly ionized metal plasmas, from which metallic ions are extracted. MeVVAs have been developed for use as sources of metallic ions. A generic MeVVA consists of a series of electrodes, usually concentric, that are separated by ceramic insulators. The usual configuration consists of a solid electrode, from which material is vaporized, followed by a trigger electrode, an anode, a suppressor, and an extractor. Triggering of the vacuum arc is 
accomplished by applying a short high voltage pulse between the trigger electrode and the cathode across an insulating surface. Discharge occurs due to formation of "cathode spots", micron-sized spots on the cathode surface characterized by extremely high current densities. Cathode material is vaporized and ionized and a plasma plume forms. Arc currents of 10s to 100s of amperes is the usual range of discharge currents for MeVVA ion sources, depending on their size and application. These ion sources vary in size, extracted current, and pulse length. The beam extraction area ranges from $0.05 \mathrm{~cm}^{2}$ to $2000 \mathrm{~cm}^{2}$, while the maximum extracted current varies among the sources from $10 \mathrm{~mA}$ to $3.5 \mathrm{~A}$. Most MeVVAs operate with pulse lengths of 0.1 to a few msec, although there are a few MeVVAs that operate dc. A good general reference on MeVVAs is Ian Brown's (the original developer of MeVVAs) review in The Physics and Technology of Ion Sources, edited by I.G. Brown (Wiley, New York, 1989), p. 331.

\section{MeVVA Source of Primary Ions for SuperEBIS}

From the large variety of MeVVA sources, we chose a miniature version called MicroMeVVA II. As shown in Figure 1 and 2, the source has an overall length of about $10 \mathrm{~cm}$, a diameter of roughly $2 \mathrm{~cm}$, and it weighs about $100 \mathrm{~g}$. Based on our needs of a less than 100 microsecond long pulse of a few microAmperes of primary ions, this MeVVA version seems very well suited for our initial tests of SuperEBIS; but, adaptation of an improved MeVVA version is planned for the future.

There are a number of possible electrical circuits that can be used to operate this source. We have been using the configuration that is schematically represented in Figure 3.

This source has been used to generate aluminum, titanium, and uranium beams. Typical operating parameters of our source are as follows. On a test stand in which the pressure in the vacuum chamber was about $1 \times 10^{-5}$ Torr, the open circuit voltage (pre-breakdown) was about 300 volts and the arc burning 
voltage was about 25 Volts. The arc current was adjusted to be usually in the range of 100-150 Amperes.

With a new cathode, the trigger voltage needed to be pushed to our maximum capability of $20 \mathrm{kV}$. After a few pulses, the trigger voltage can be lowered. Eventually, after thousands of pulses, the trigger voltage can be reduced to only a few hundred volts.

Obtaining a very accurate measurement of the total current output from a MeVVA requires a good current transformer or a special Faraday cup, of which we had neither. The reason for this stems from beam divergence and large emission of VUV from the MeVVA discharge that causes large residual ionization. Nevertheless, based on current measurements on some plates, a lower limit estimate for the current output from our MeVVA is $10 \mathrm{~mA}$. This is orders of magnitude more than what SuperEBIS needs.

After the MeVVA was installed on SuperEBIS, where the base pressure was no higher than $1 \times 10^{-7}$ Torr, it was much more difficult to start with a new cathode. However, after putting some pencil marks on an insulator, the source was successfully deployed on SuperEBIS.

Charge state distributions of the generated titanium and uranium beams have been measured using a time-of-flight spectrometer (which was fabricated here at BNL based on an invention by Mamyrin and is described in AGS/AD/Tech. Note No. 390; original reference is B.A. Mamyrin, et al., Sov. Phys.-JEPT, 37, 45 (1973)). Figures 4 and 5 show the charge state distribution for titanium and uranium, respectively. 


\section{Figure Captions}

Figure 1. Assembled appearance of MeVVA. The ion beam exits from the left.

Figure 2. Cross section (not to scale) of the MeVVA. All electrodes are made out of stainless steel.

Figure 3. A diagram of the electrical system used to operate MeVVA.

Figure 4. Charge state distribution of $\mathrm{Ti}$ ions. Chopper pulse width $=$ (a) $2 \mu \mathrm{s}$ and (b) $1 \mu \mathrm{s}$. The vertical arrow marks the start of the chopper pulse. Horizontal sensitivity was $5 \mu \mathrm{s} / \mathrm{div}$. Ion extraction voltage was $\approx 1.2 \mathrm{kV}$.

Figure 5. Charge state distribution for Uranium ions. Horizontal sensitivity was $10 \mu \mathrm{s} /$ div. Approximately $1 \mathrm{kV}$ extraction voltage. 


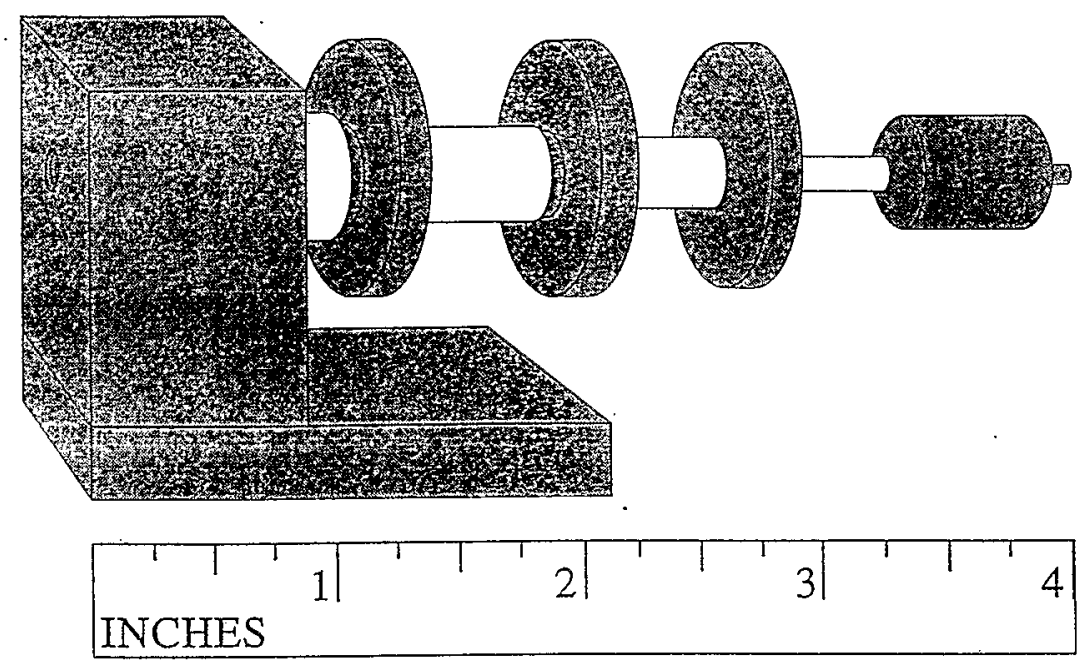

Figure 1.

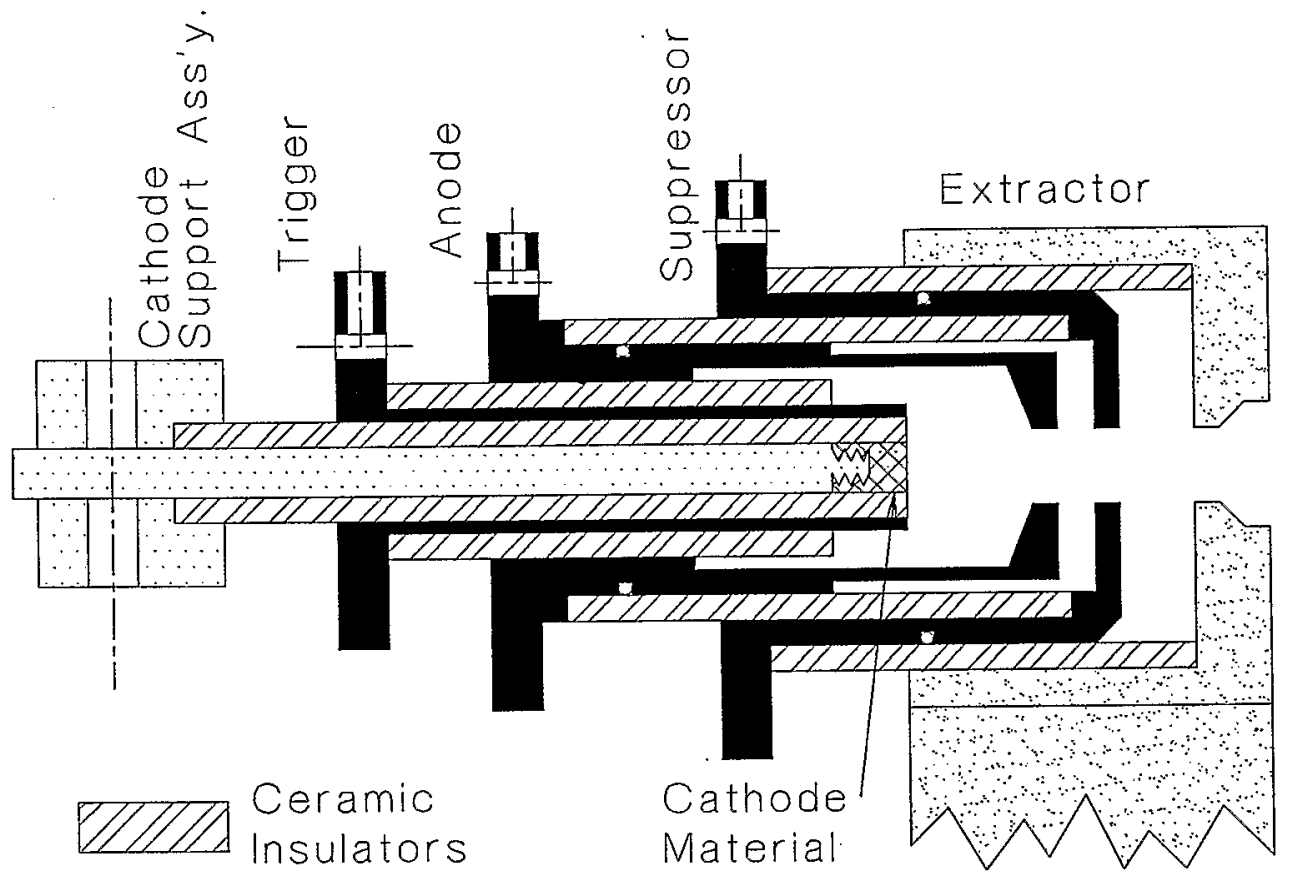

Figure 2. 


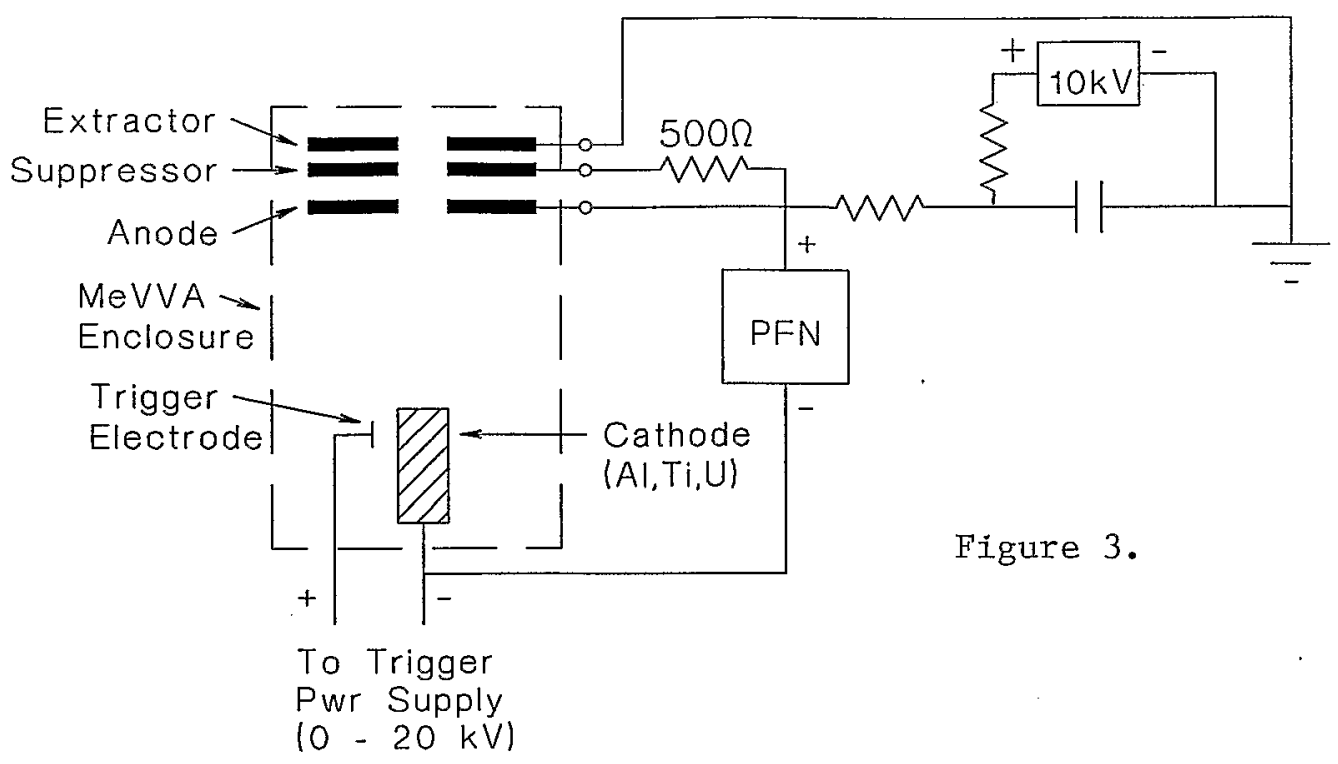

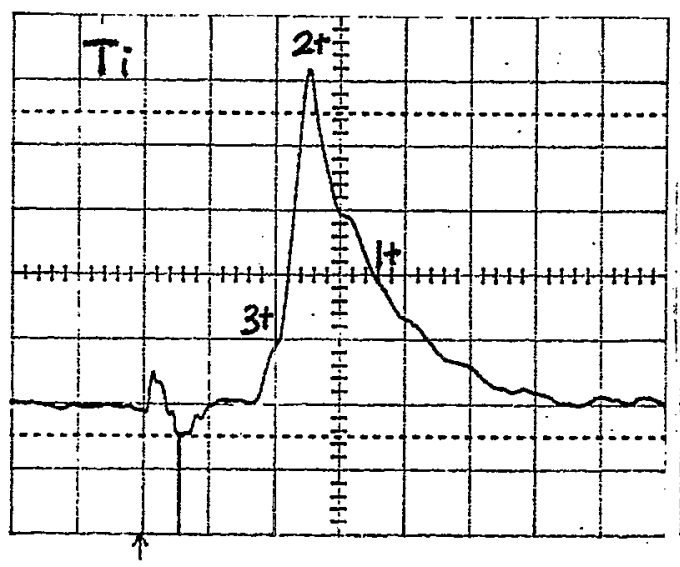

(a)

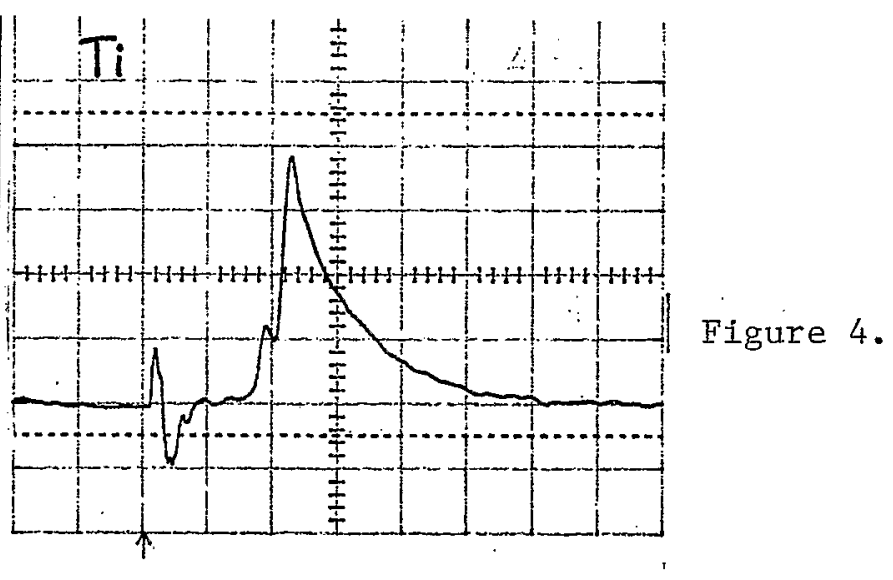

(b)

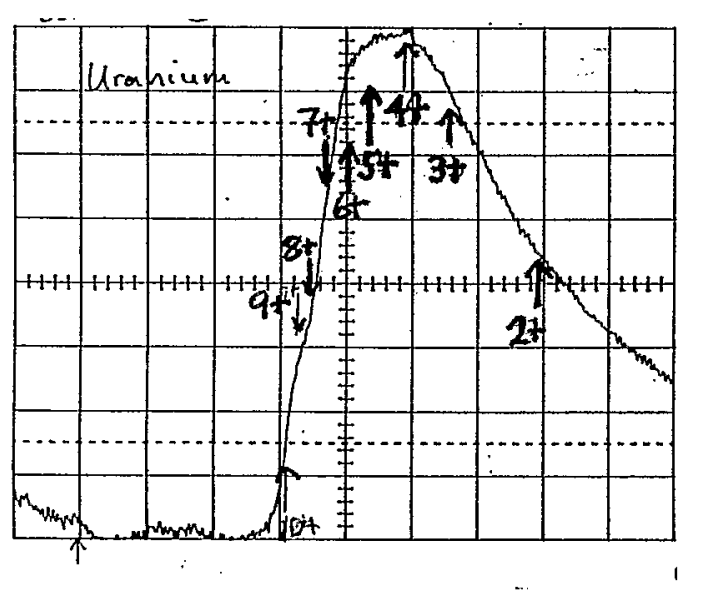

Figure 5. 